\title{
Taylor relaxation and $\lambda$ decay of unbounded, freely expanding spheromaks
}

\author{
J. Yee and P. M. Bellan ${ }^{\text {a) }}$ \\ California Institute of Technology, Laboratory of Applied Physics, MS 128-95, Pasadena, California 91125
}

(Received 4 February 2000; accepted 22 May 2000)

\begin{abstract}
A magnetized coaxial gun is discharged into a much larger vacuum chamber and the subsequent evolution of the plasma is observed using high speed cameras and a magnetic probe array. Photographic results indicate four distinct regimes of operation, labeled I-IV, each possessing qualitatively different dynamics, with the parameter $\lambda_{\text {gun }}=\mu_{0} I_{\text {gun }} / \Phi_{\text {bias }}$ determining the operative regime. Plasmas produced in Regime II are identified as detached spheromak configurations. Images depict a donut-like shape, while magnetic data demonstrate that a closed toroidal flux-surface topology is present. Poloidal flux amplification shows that Taylor relaxation mechanisms are at work. The spatial and temporal variation of plasma $\lambda=\mu_{0} J_{\phi} / B_{\phi}$ indicate that the spheromak is decaying and expanding in a manner analogous to a self-similar expansion model proposed for interplanetary magnetic clouds. In Regime III, the plasma is unable to detach from the gun due to excess bias flux. Analysis of toroidal and poloidal flux as well as the $\lambda$ profile shows that magnetic flux and helicity are confined within the gun for this regime. (c) 2000 American Institute of Physics. [S1070-664X(00)01709-2]
\end{abstract}

\section{INTRODUCTION}

Taylor's relaxation model ${ }^{1,2}$ has been successfully applied to many fusion related plasmas, especially spheromaks and reverse field pinches. However, there is substantial evidence suggesting that relaxation concepts apply well beyond the bounds of the fusion arena. Numerous solar, space, and astrophysical plasmas exhibit helical force-free magnetic fields suggestive of minimum energy Taylor equilibria; examples include interplanetary magnetic clouds, ${ }^{3,4}$ solar prominences, ${ }^{5}$ the earth's magnetotail, ${ }^{6}$ and astrophysical jets. $^{7}$

The laboratory experiment described in this paper employs a magnetized coaxial spheromak gun which injects plasma into a very large stainless steel vacuum vessel. The large size of the vessel effectively removes the fluxconserving boundary common to all previous spheromak experiments and so simulates the vacuum boundary typical of space plasmas.

The aim of this project is to characterize the formation and evolution of an unconfined spheromak produced by a coaxial gun. Details of the formation and reconnection process are examined in order to determine the extent to which Taylor relaxation occurs. Departures from the minimum energy state are quantified and the concepts of helicity conservation, flow, and injection threshold are tested.

The experiment discussed here resembles and extends an experiment performed nearly four decades ago by Alfvén, Lindberg, and Mitlid, ${ }^{8}$ where plasma generated by a coaxial gun was found to exhibit interesting helical features and flux amplification. However, this early effort was undertaken without the benefit of modern diagnostic techniques, computational analysis tools, and, most importantly, insight drawn from the Taylor relaxation model. Furthermore, Alfvén, Lindberg, and Mitlid's device was fired into an insulating (glass) container, which was only moderately larger than the

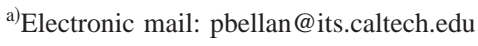

gun, and thus the plasma in their experiment was not permitted to freely expand without interacting with boundaries.

\section{APPARATUS AND DIAGNOSTICS}

Figure 1 shows the experimental setup. A magnetized coaxial plasma gun is mounted on the end wall of a larger cylindrical vacuum chamber. The size of the vacuum chamber provides generous optical access to view the plasma dynamics and also avoids plasma-wall interactions, so that boundary conditions appropriate for a freely expanding spheromak are achieved.

The inner coaxial gun electrode is a $6.4 \mathrm{~cm}$ diam. tantalum sheathed copper tube and the outer electrode is a $16 \mathrm{~cm}$ inner diameter copper tube which is spray-coated with tungsten. The gun is powered by a $120 \mu \mathrm{F}$ ignitron-switched capacitor bank which was operated in a range of 4-8 kilovolts. Bias fluxes $\Phi_{\text {bias }}$ of up to $7 \mathrm{mWb}$ are produced by a solenoid coil. Vacuum is maintained by a $14^{\prime \prime}$ APD Cryogenics cryopump, providing $\mathrm{a} \approx 2 \times 10^{-7}$ Torr base pressure. A Varian sorption pump is used for rough pumping of the chamber from atmosphere. This oil-free vacuum system ensures a high level of cleanliness.

All timing trigger signals are provided by a custom-built programmable, fiberoptic-coupled timing module. The operation sequence of a typical plasma discharge proceeds as follows: A gas puff valve is triggered $23 \mathrm{~ms}$ after the bias flux bank. The main gun capacitor bank is fired $400 \mu$ s afterwards. Simultaneously, digitization of the various diagnostic wave forms is initiated. Finally, after an adjustable time delay (typically 4-25 $\mu \mathrm{s}$ ), a gated Princeton Instruments intensified charge-coupled device (ICCD) camera is triggered.

A magnetic probe array was used to measure the local magnetic field in the plasma. The probe array consists of a linear array of small magnetic pickup loops. The probe array is located on a Teflon form, which is threaded into a 0.25 inch outer diameter thin-walled (0.010 inch) stainless steel 


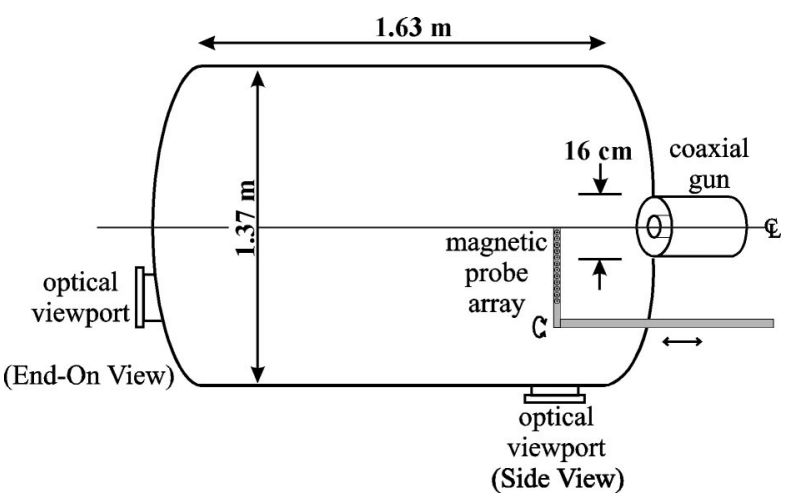

FIG. 1. Top view of coaxial gun, vacuum chamber, and magnetic probe diagnostic.

tube which is in turn sheathed in a $8 \mathrm{~mm}$ outer diameter protective, insulating ceramic tube. The stainless tube is capped on its end, and serves the dual purposes of maintaining vacuum and providing electrostatic shielding. The L-shaped magnetic probe arm passes through an O-ring vacuum seal mounted on a 2.75 inch Conflat flange. The O-ring seal allows both axial translation and azimuthal rotation. A three-dimensional volume of space can thus be probed by the array. The axial travel is restricted so that the probe array cannot be positioned any closer than $10 \mathrm{~cm}$ from the mouth of the gun.

Two coordinate systems will be used in describing locations in the vacuum chamber. The first is $(r, \phi, z)$, the natural

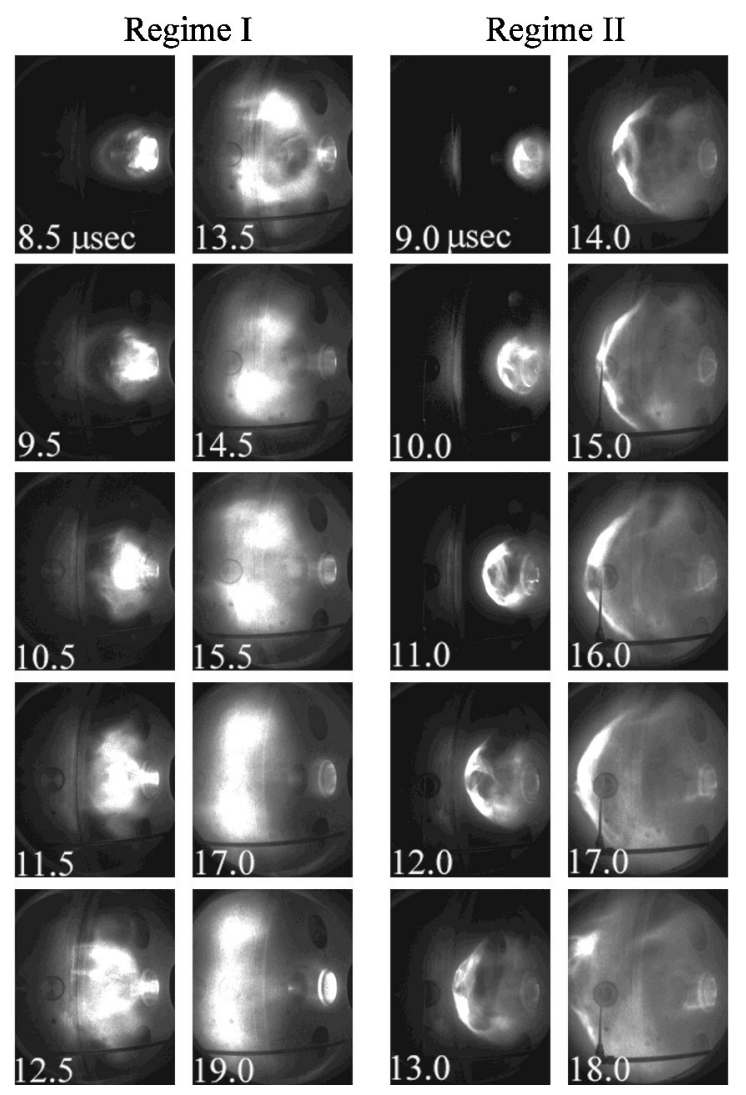

FIG. 2. Side View image sequences with gun parameters: $I_{\text {gun }}=130 \mathrm{kA}$, $\Phi_{\text {bias }}=0.4 \mathrm{mWb}$ (Regime I) and $I_{\text {gun }}=130 \mathrm{kA}, \Phi_{\text {bias }}=2 \mathrm{mWb}$ (Regime II). cylindrical coordinate system of the coaxial gun electrodes. The axis of this system is coincident with the geometrical axis of the gun and $z$ is measured from the muzzle of the gun (end of outer electrode). The second system is $\left(r^{\prime}, \phi^{\prime}, z^{\prime}\right)$, the natural cylindrical coordinate system of the magnetic probe array. The axis is the rotational axis of the probe array, parallel to the axis of the unprimed system but offset from it. The axial coordinate $z^{\prime}$ is also measured from the same origin as the unprimed system, so that $z^{\prime}=z$. (For example, the inaccessible entrance region is located at $0<z^{\prime}<10 \mathrm{~cm}$.) The $\phi^{\prime}=0$ direction is defined to point towards the gun axis, while the $\phi=0$ direction is defined to point towards the probe axis.

\section{CLASSIFICATION OF OPERATING REGIMES}

The camera is a powerful diagnostic tool because it provides a simple means of obtaining direct information about the gross structure of the plasma, which may reveal the underlying structure of the magnetic field. In order to characterize gun operation photographically, the gun was operated over a wide range of parameters. During the course of these parameter-space surveys, qualitative plasma evolution was observed to fall into one of four categories, which are referred to as Regimes I-IV. Sample composite movie sequences of these regimes were assembled from multiple identical plasma shots and are presented in Figs. 2 and 3. These pictures were taken with the camera positioned to image the plasma from the Side View (see Fig. 1). The vacuum port of the plasma gun is visible as an upright oval at the right of each image. The muzzle of the gun is located near this position, and plasma is ejected from the gun across the field of view, from right to left.

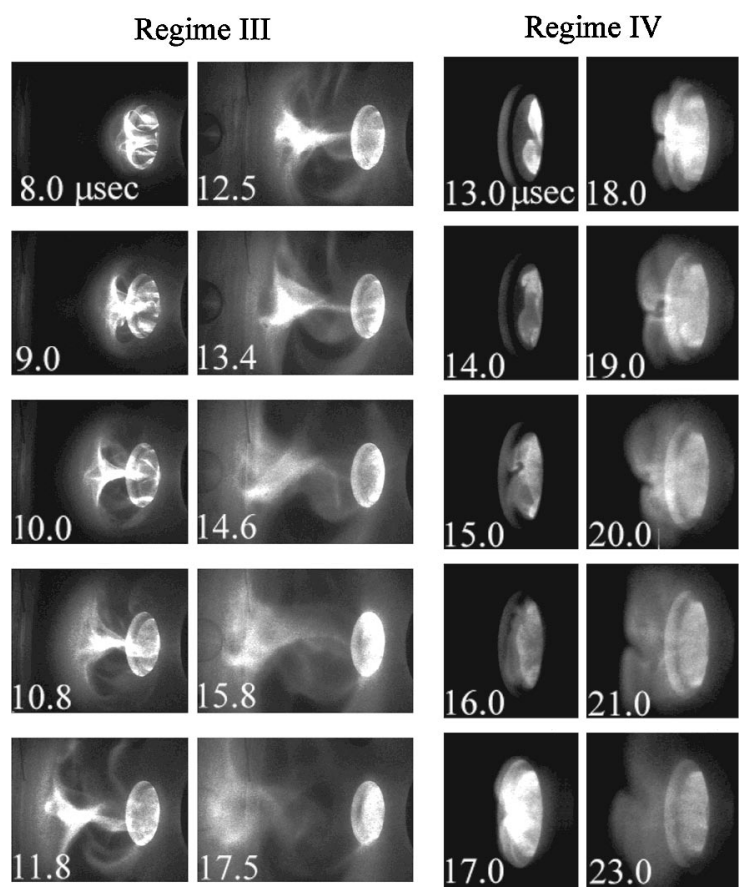

FIG. 3. Side View image sequences with gun parameters: $I_{\text {gun }}=130 \mathrm{kA}$, $\Phi_{\text {bias }}=6 \mathrm{mWb}$ (Regime III) and $I_{\text {gun }}=90 \mathrm{kA}, \Phi_{\text {bias }}=6 \mathrm{mWb}$ (Regime IV). 


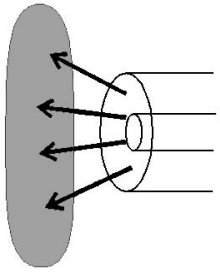

\section{Regime I}

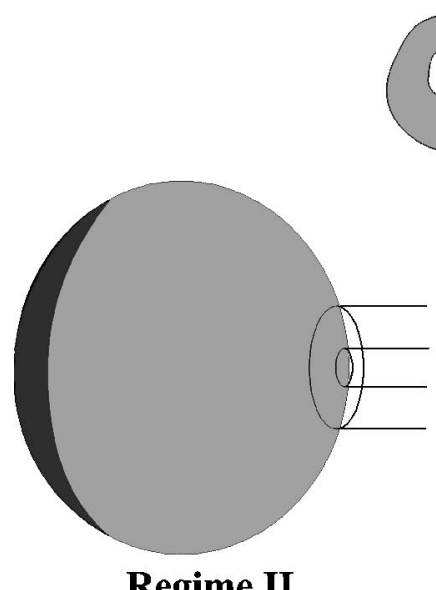

Regime II
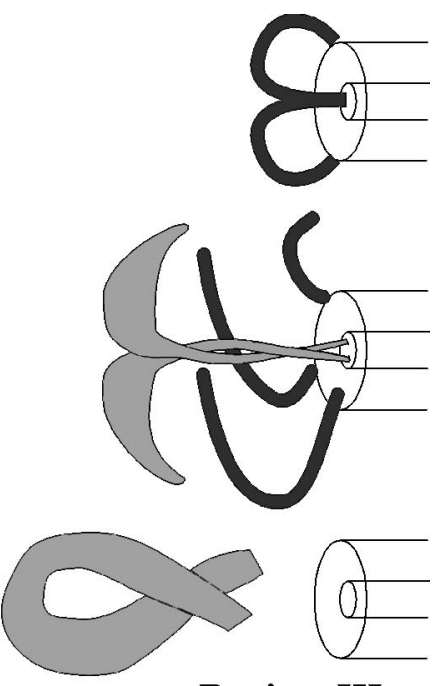

Regime III

FIG. 4. Representative sketch of plasma dynamics in the four Regimes.

In Regime I (Fig. 2), the plasma remains fairly homogeneous throughout the discharge, exhibiting no fine structure. The plasma exits the gun as an amorphous blob, as sketched in Fig. 4. The gun parameters are $I_{\text {gun }}=130 \mathrm{kA}$ and $\Phi_{\text {bias }}$ $=0.4 \mathrm{mWb}$ for this case, corresponding to a nearly unmagnetized discharge, so that little helicity content is present and the applied bias field does not impede the plasma. (In this case, the bias flux is provided by residual magnetization in the iron magnetic circuit.)

In Regime II (Fig. 2), the plasma emerges with a spherical shape which further expands over time. The well-defined left edge is especially bright and sporadically contains filamentary structure. The general appearance is sketched in Fig. 4. Here, the gun parameters are $I_{\text {gun }}=130 \mathrm{kA}$ and $\Phi_{\text {bias }}=2$ $\mathrm{mWb}$. Evidence will be presented later showing that a spheromak-like state is generated in this regime.

In sharp contrast to the other regimes, the plasma in Regime III (Fig. 3) contains a wealth of fine structure. Of particular interest are the distinct central column (twisted) and helical outer loops. These features evolve through a sequence of stages and Fig. 4 sketches the appearance of the plasma for this regime. The gun is operated at $I_{\text {gun }}=130 \mathrm{kA}$ and $\Phi_{\text {bias }}=6 \mathrm{mWb}$ for these images. Analysis of magnetic data presented below will show that, in this regime, the plasma is unable to detach from the gun due to excess bias flux.

Finally, in Regime IV (Fig. 3), the plasma emerges very slowly from the gun and decays away at the mouth of the

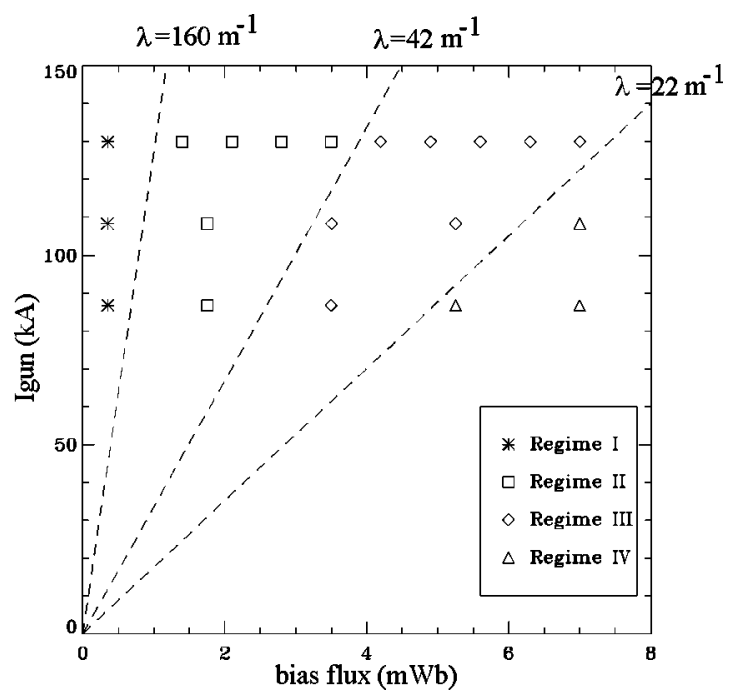

FIG. 5. Survey of Regime classification vs gun current and bias flux. Dashed lines indicate $\lambda=160,42$, and $22 \mathrm{~m}^{-1}$.

gun. The high bias flux dominates plasma dynamics and confines the plasma, as sketched in Fig. 4. The gun parameters in this regime are $I_{\text {gun }}=90 \mathrm{kA}$ and $\Phi_{\text {bias }}=6 \mathrm{mWb}$.

A key parameter governing the behavior of a spheromak gun is $\lambda_{\text {gun }}=\mu_{0} I_{\text {gun }} / \Phi_{\text {bias }}$. The boundaries of the four regimes were found to be associated with the gun current and bias flux parameters. This dependence is shown in Fig. 5. Each point corresponds to a series of photos, and gives a subjective determination of the appropriate regime classification. Approximate $\lambda_{\text {gun }}$ boundaries between regimes are also shown.

\section{REGIME II: EXPERIMENTAL DATA}

Regimes I and IV correspond to less interesting extreme cases where the plasma is either unaffected by, or dominated by the bias flux. On the other hand, the plasmas in Regimes II and III exhibit helical structure expected to be associated with magnetic helicity. For this reason, in the remainder of this paper we focus on Regimes II and III. In this section, data obtained in Regime II is discussed.

\section{A. Current and voltage characteristics}

The current and voltage wave forms with the gun operated with nominal settings in Regime II $\left(I_{\text {gun }}=130 \mathrm{kA}\right.$, $\Phi_{\text {bias }}=2 \mathrm{mWb}$ ) are displayed in Fig. 6. The gun bank ignitron switch breaks down and enters conduction mode at $t$ $=1 \mu \mathrm{s}$, followed by plasma breakdown $\sim 0.3 \mu$ s afterwards. The plasma behaves as a primarily reactive load so that the circuit exhibits underdamped oscillations. The current rises to its peak value of $130 \mathrm{kA}$ at $6.5 \mu \mathrm{s}$ and reverses polarity at $14.5 \mu \mathrm{s}$. Since the helicity injection rate is $2 \Phi V$ where $V$ is the voltage across the electrodes, ${ }^{9}$ helicity injection terminates when the voltage reverses polarity at $9.5 \mu \mathrm{s}$, and after this time one might expect that helicity is extracted from the plasma volume. However, direct measurements of plasma current described below suggest that this model is exces- 


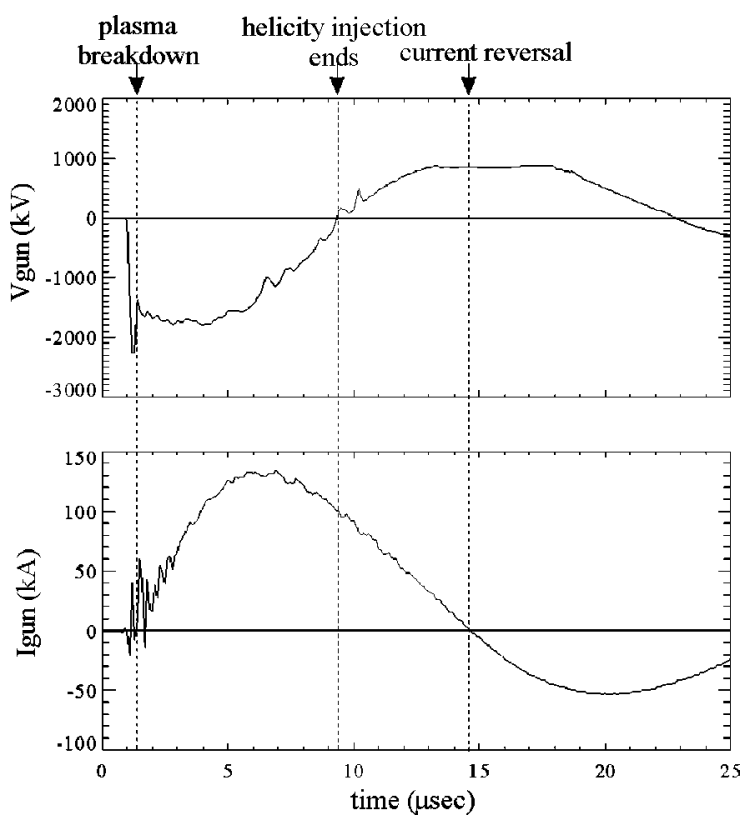

FIG. 6. Sample gun current and voltage traces for gun operation at the nominal settings in Regime II.

sively simplistic. The data show that, even in the high bias flux (e.g., Regime III) cases where the plasma remains attached to the gun, plasma current polarity reversal does not occur upon gun current polarity reversal. This implies the presence of residual plasma which short-circuits the gun and may reduce the period of helicity injection.

\section{B. End-on camera images}

In addition to the Side-View pictures presented earlier, information can also be extracted from images taken from the End-On View, shown in Fig. 7. These images take the form of a ring encircling the geometric axis of the gun. The ring first appears at $11 \mu$ s and gradually expands in diameter.

A significant amount of irreproducibility is exhibited in these pictures. In the End-On Views, some images show filaments of material emerging from the central electrode and twisting counter-clockwise around the geometric axis. Some images show multiple strands encircling the axis. In some images, the ring of material is not well centered on the axis. Nevertheless, a clear ring-shaped structure is always visible emerging from the gun.

Correlation of the Side View and End-On View images provides evidence of plasma encircling the gun geometric axis. It is not clear from these images whether a distinct spheromak possessing the characteristic nested toroidal flux surface magnetic topology is in fact ejected. In order to obtain direct and quantitative information about the magnetic structure of the plasma, the magnetic probe array was used.

\section{Magnetic traces}

A sample of data obtained using the probe array is shown in Fig. 8 for a shot taken with nominal gun parameters. For this data, the probe was positioned at $z=30 \mathrm{~cm}$
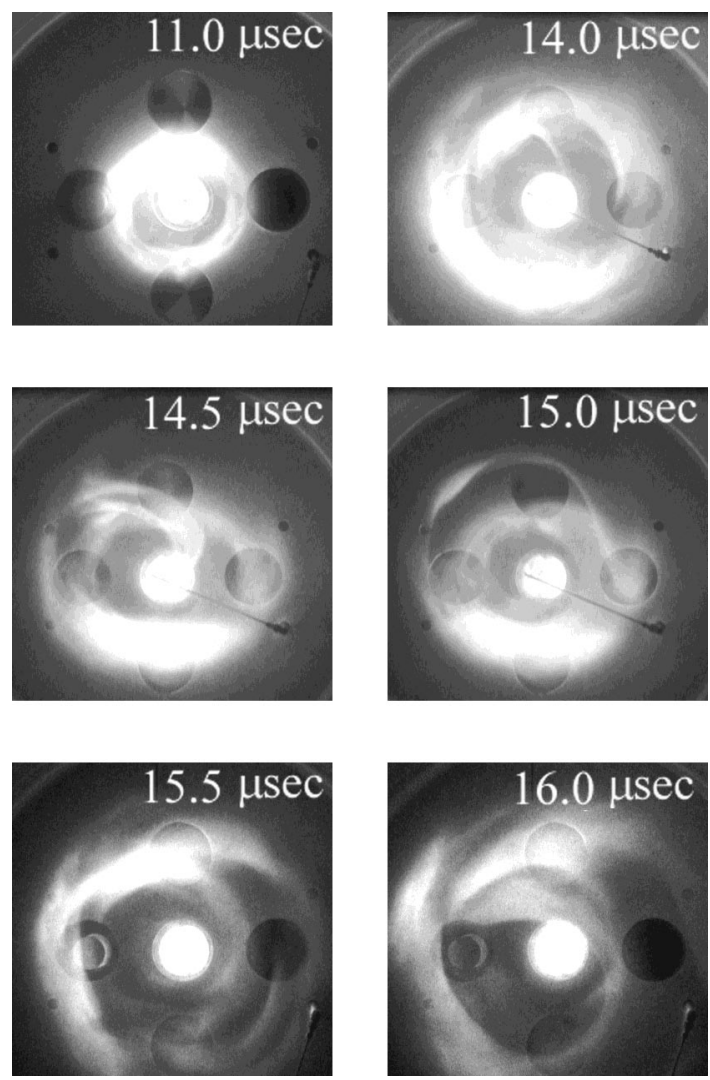

FIG. 7. Selected End-On views with gun operated at nominal settings in Regime II.

and rotated so that it lay in a gun poloidal plane $\left(\phi=\phi^{\prime}\right.$ $=0$ ). For each magnetic field direction, the time dependence is measured at 12 radial arm locations.

Most of the magnetic activity takes place between $t$ $=12 \mu \mathrm{s}$ and $t=20 \mu \mathrm{s}$. During this time period, $B_{\phi}$ is typically unipolar and positive, while $B_{z}$ is negative near the axis and positive further away from the axis. $B_{r}$ oscillates through $\sim 1.5$ cycles. This behavior will be shown below to be consistent with a spheromak configuration.

A smaller secondary event occurs at $t=28-35 \mu \mathrm{s}$, and is likely associated with an additional plasma ejection produced by the negative gun current peak at $t \simeq 20.5 \mu$ s (see Fig. 6). We will restrict discussion to the primary event at $t$ $=12-20 \mu \mathrm{s}$.

Magnetic probe data was obtained at a variety of axial and azimuthal positions. Compiling data from multiple shots in order to develop a complete picture of magnetic configuration proved unfeasible due to shot-to-shot irreproducibility. Although shots with identical gun parameters generated qualitatively similar magnetic wave forms, characteristics such as magnitudes and time offsets were often inconsistent. A propagation-based technique was developed in order to overcome this problem.

\section{PROPAGATION INFERENCE TECHNIQUE}

\section{A. Propagation rate}

The propagation of the expanding front of the ejected plasma is shown in Fig. 9. As the axial position of the mag- 

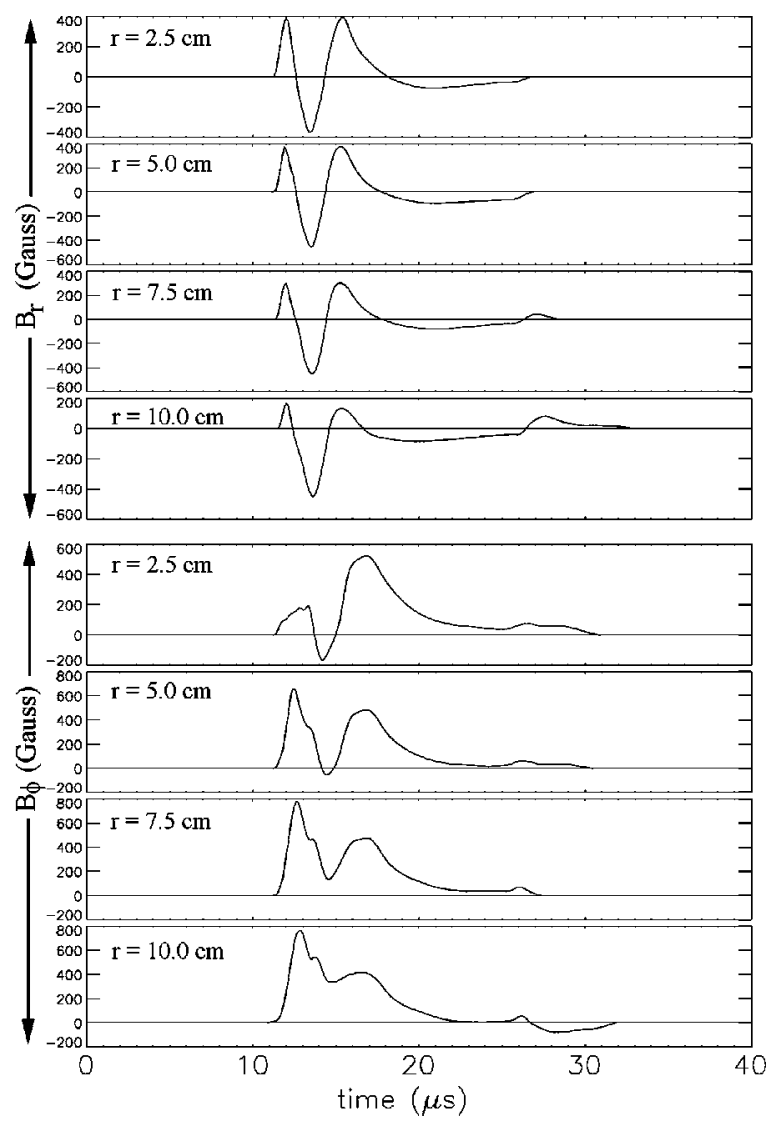

FIG. 8. Sample magnetic probe data with gun operated at nominal settings in Regime II. Magnetic probe is positioned at $z=30 \mathrm{~cm}, \phi=0$. Top four plots show $B_{r}$ at four radial positions; bottom four plots show $B_{\phi}$.

netic probe is varied (with azimuthal position fixed at $\phi$ $=0$ ), the time at which magnetic field is first detected changes. In this plot, this time is determined from the instant at which the $\phi$ component of the field at radial position $r$ $=7.6 \mathrm{~cm}$ exceeds an arbitrary threshold value of 100 Gauss, and is represented by an asterisk. (The arbitrary level was

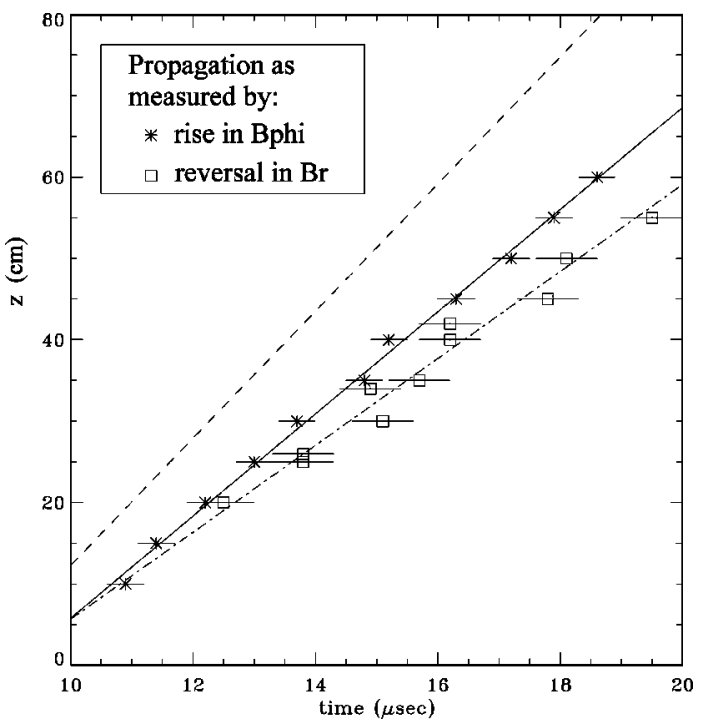

FIG. 9. Position of the expanding plasma front vs time. Data points correspond to magnetic data. Dashed line shows propagation of optical image. chosen to be significantly above the noise level, but well below the peak values of up to $1.5 \mathrm{kG}$.) A linear regression of these data points produces a propagation speed of 8.6 $\mathrm{cm} / \mu \mathrm{s}$ and fits the data remarkably well.

An alternative method of calculating the propagation speed is based on the first positive to negative transition in $B_{r}$ (cf. Fig. 8). As will be seen below, this reversal point is associated with the magnetic axis of the spheromak. The times at which the $B_{r}$ probe measurement at $r=7.6 \mathrm{~cm}$ reverse sign are indicated by squares. Linear regression yields a slope of $8 \mathrm{~cm} / \mu \mathrm{s}$, but fits the data less well than the previous calculation. For reference, a camera-based propagation rate is represented by a dashed line in the plot.

\section{B. Propagation inferred data}

In the following, we make the conjecture that the magnetic field structure remains fixed in the plasma and is merely convected along at the propagation rate in the $z$ direction. This property is suggested by the qualitative consistency of magnetic wave forms at varying axial probe positions. This conjecture provides a powerful means for examining the magnetic configuration using only one discharge. The $z$-dependence of $\mathbf{B}$ is entirely inferred from the time dependence of the wave forms.

The conjecture is applied to magnetic data as follows: The magnetic field measured by the probe at position $z_{\text {probe }}$ is $\mathbf{B}\left(r, z_{\text {probe }}, t\right)$. If convection at a speed $v_{\text {prop }}$ is assumed, then the time-dependent field measured at an arbitrary point is

$$
\mathbf{B}(r, z, t)=\mathbf{B}\left(r, z_{\text {probe }}, t-\left(z-z_{\text {probe }}\right) / v_{\text {prop }}\right),
$$

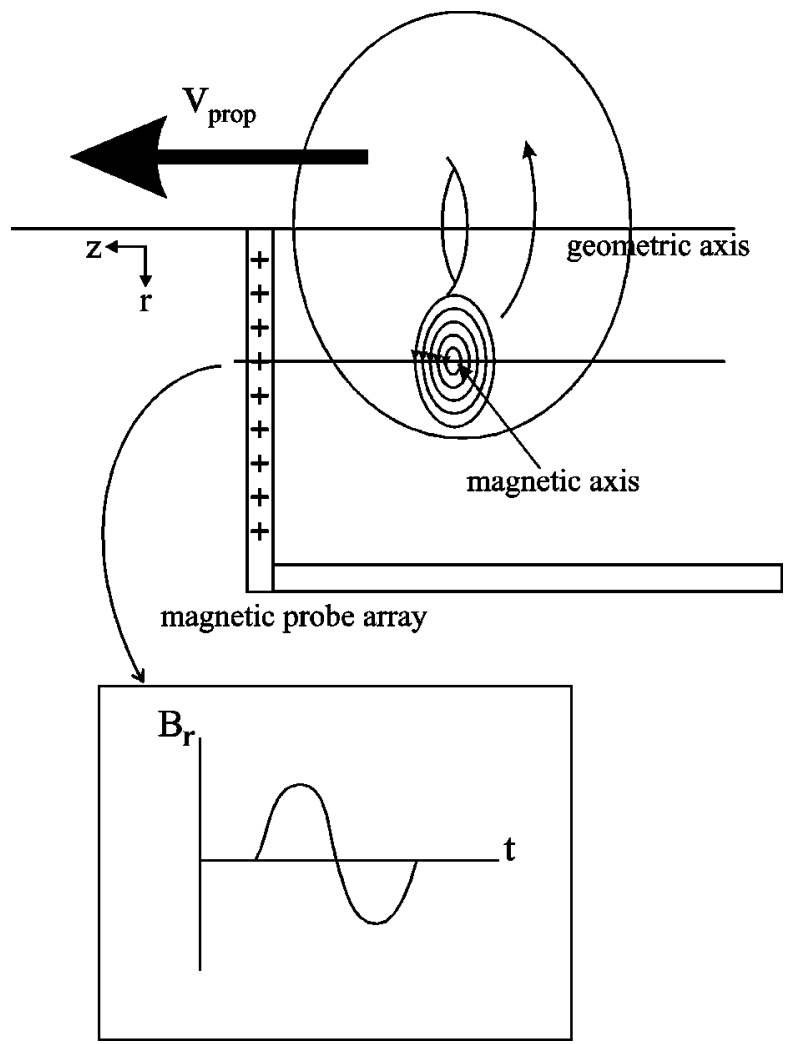

FIG. 10. Sketch of the SSPI method. 
and can be directly obtained from the field measured at the probe.

This Single Shot Propagation Inference (SSPI) method is shown schematically in Fig. 10. The donut-shaped spheromak travels from right to left through the stationary magnetic probe array. The signal detected by a $B_{r}$ probe at one probe position (which is aligned with the magnetic axis of the spheromak) is shown versus time. It is also evident from this plot that the positive to negative transition of $B_{r}$ is associated with the passage of the magnetic axis. For probe locations near the gun, $z \leqslant 30 \mathrm{~cm}, 1.5$ cycles of $B_{r}$ are observed (e.g., Fig. 8); the trailing 0.5 cycle indicates that some magnetic flux remains attached to the gun.

Figure 11 shows a magnetic field plot constructed based on the above technique from the magnetic data presented in Fig. 8 (with the probe positioned at $z=30 \mathrm{~cm}$ and $\phi=0$ ). In this gun coordinate poloidal slice, the poloidal field vectors are shown in the lower plot and the toroidal field appears as a contour plot in the upper plot. The coaxial gun electrode system is shown schematically, with the gun vertical (but not horizontal) dimension to scale. The actual probe position is indicated by an arrow on the horizontal axis. It is important to note that the magnetic field has been measured only at this point in space. Data to the left (increasing $z$ ) of this location correspond to data taken at earlier times in the discharge (relative to $t=12 \mu \mathrm{s}$ ) and data to the right correspond to data taken at later times. Thus, the data is exact at the probe location and may be expected to increasingly become an approximation away from this location (a propagation rate of $8 \mathrm{~cm} / \mu$ s was used in this calculation).

Using this SSPI technique, the spheromak configuration becomes immediately obvious. The vortex-like swirl pattern in the poloidal direction combined with the peak of negative polarity in the toroidal field are clearly consistent with the spheromak magnetic topology. Within the context of this propagation viewpoint, there is a close connection between the positive to negative transition in $B_{r}$ and the location of the magnetic axis. Trailing behind the spheromak, an $\mathrm{X}$-point is also clearly visible, indicating that the reconnection region arrives at $z=30 \mathrm{~cm}$ approximately $1.5 \mu$ s later.

\section{Consistency check}

The validity of the SSPI technique may be tested by comparing the fields inferred from several different shots fired with the probe located at different axial positions. This self-consistency check is shown in Fig. 12 for $t=12 \mu \mathrm{s}$. Ideally, the inferred field structure would be independent of probe location. Instead, the plots are qualitatively similar, but exhibit some distinguishing characteristics. The vortex pattern detected when the probe is at $z=10 \mathrm{~cm}$ seems to be less well-formed and smaller than when the probe is at $z=40 \mathrm{~cm}$. This behavior is consistent with expansion and possible relaxation of the spheromak. The X-point reconnection region also seems to disappear when the probe is at $z>20 \mathrm{~cm}$, indicating that the $\mathrm{X}$-point does not propagate beyond $\sim 20$

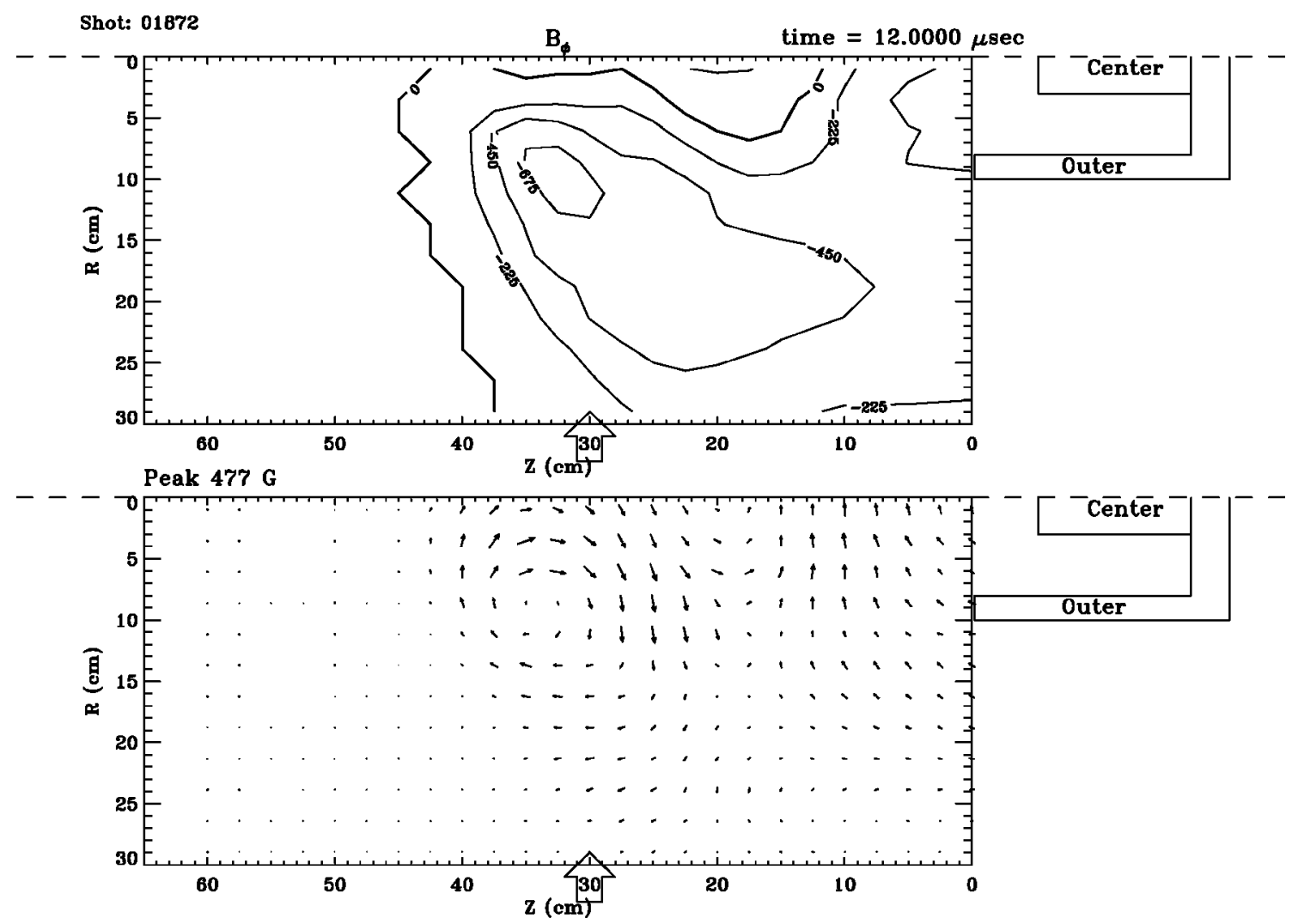

FIG. 11. SSPI magnetic data. Top plot is contour plot of $B_{\phi}$. Bottom plot is a vector field plot of $B_{r}, B_{z}$. Maximum vector length corresponds to the peak value (477 Gauss). The arrow on the horizontal axis $(30 \mathrm{~cm})$ indicates the actual position of the magnetic probe. 


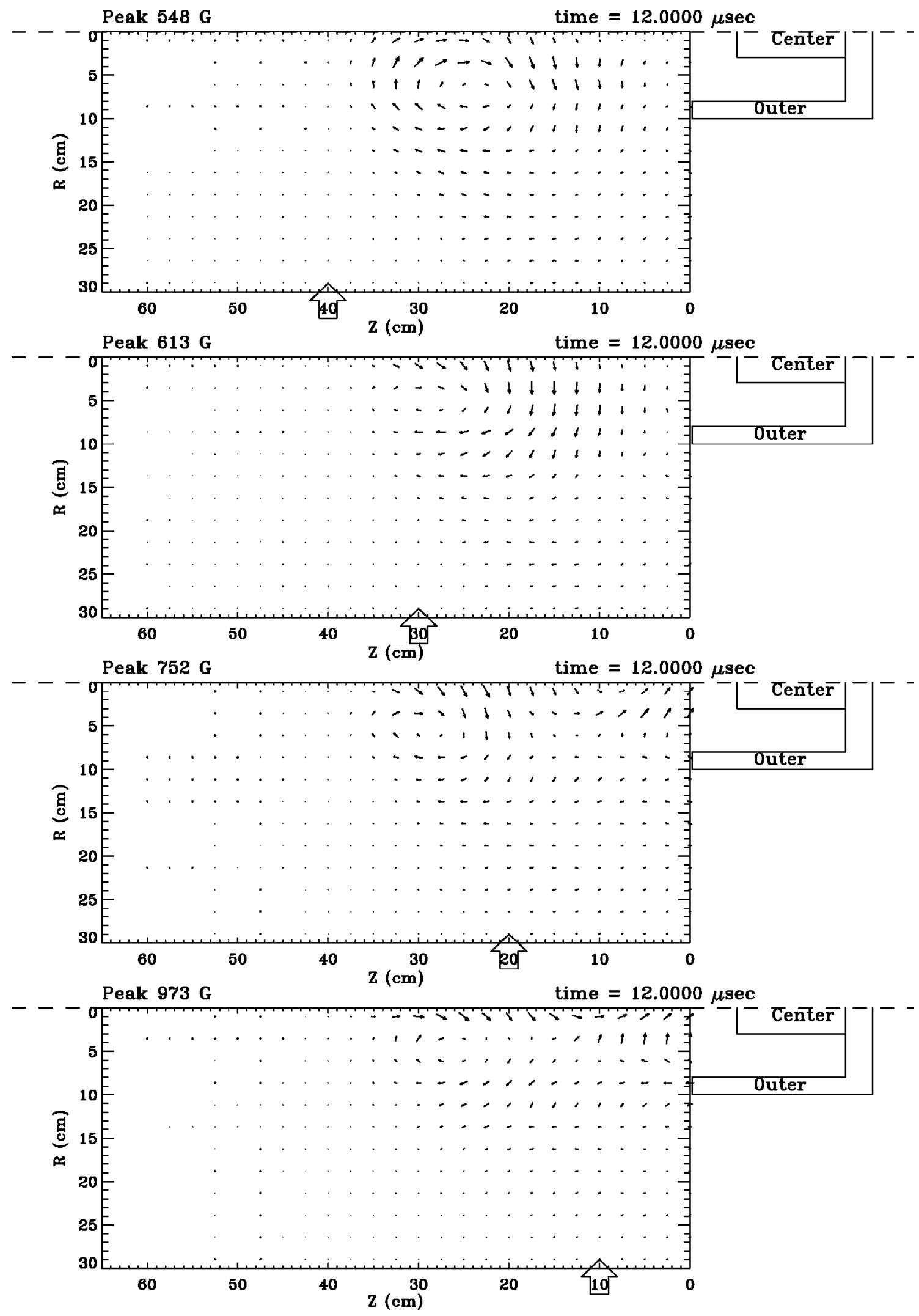

FIG. 12. Consistency check: Several SSPI field plots from data taken at differing probe locations for $t=12 \mu$ s.

$\mathrm{cm}$. Also evident from the figure is a gradual reduction in peak field magnitude at increasing distances from the gun. With these caveats, it is clear that this technique provides a good picture of field structure. This test also supports the calculated value for the propagation rate $(8 \mathrm{~cm} / \mu \mathrm{s})$.

\section{Reproducibility}

It is interesting to examine the shot-to-shot irreproducibility using the SSPI technique. Figure 13 shows SSPI measurements of several different shots having the same gun 


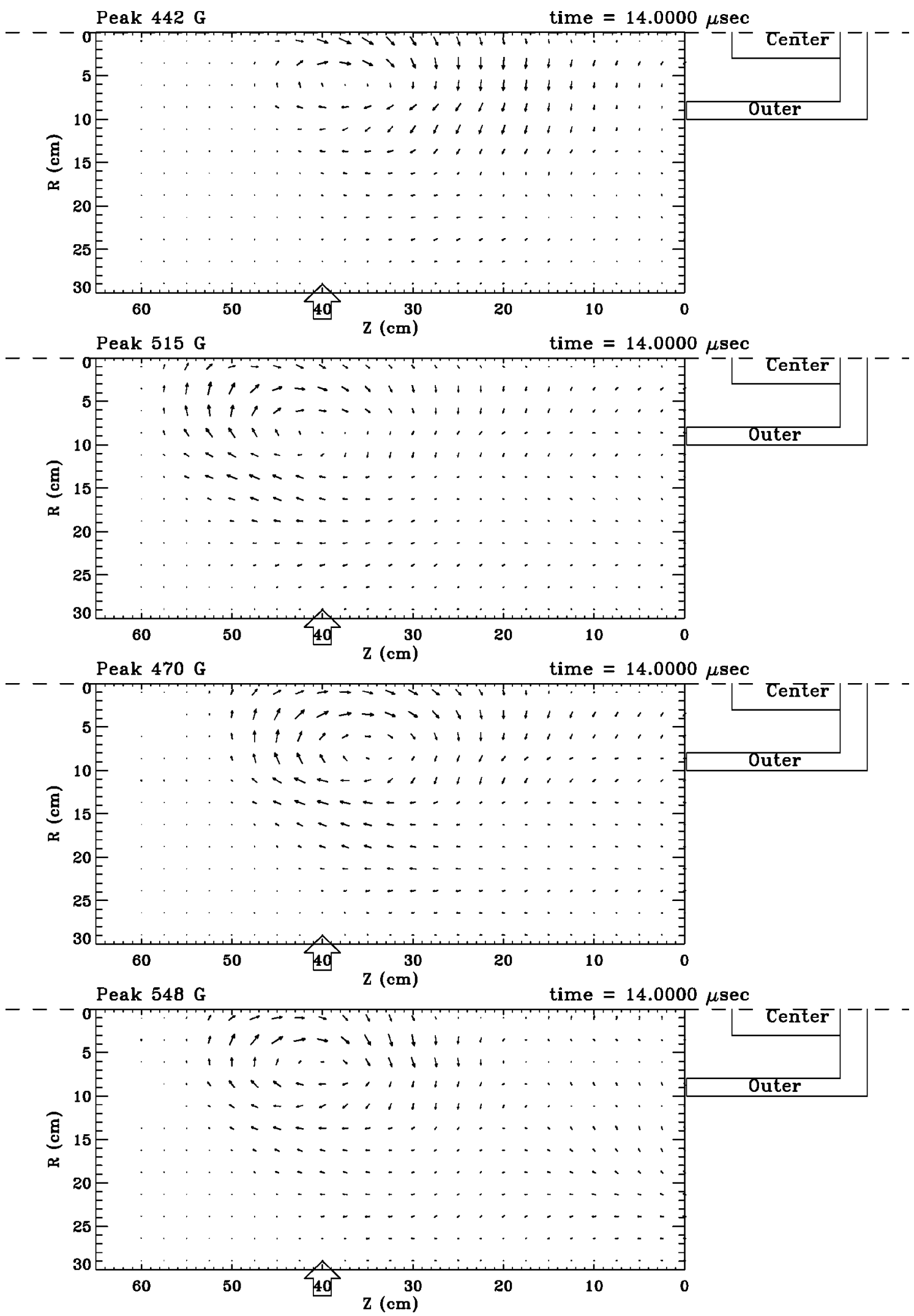

FIG. 13. Reproducibility check: Several SSPI vector field plots of plasma discharges with identical parameters.

parameters and probe position. Comparing the various vector field plots, it is apparent that the magnetic fields are qualitatively similar, with each exhibiting a clear spheromak poloidal vortex pattern. However, the field magnitude, uniformity, radial position, and axial position (the latter corresponding to a time offset) vary from shot to shot. The peak field magnitude and radial position are the most consistent, varying by $5 \%-10 \%$ typically. The axial position exhibits an uncer- 
tainty of $\sim 5 \mathrm{~cm}$ (or $0.6 \mu \mathrm{s}$ ). The field uniformity across the spheromak varies significantly from shot to shot, particularly in the radial direction. The radial field is in some cases higher in amplitude at the leading or trailing edges of the spheromak, whereas in other cases it is more uniformly distributed across the spheromak. In the former cases, this may be an indication of incomplete relaxation.

Reproducibility is also a factor with regards to the reconnection region. Although the $\mathrm{X}$-point is always visible with data taken at probe positions of $z_{\text {probe }} \leqslant 20 \mathrm{~cm}$, reconnection is intermittently visible for $z_{\text {probe }}=30 \mathrm{~cm}$. For example, this variability is apparent in comparing the vector field plots in Fig. 11 with the corresponding plot in Fig. 12 (also at $z_{\text {probe }}=30 \mathrm{~cm}$ ). The substantial irreproducibility observed in the details of reconnection is not surprising and is likely due to the complex nature of the reconnection process.

\section{E. Discussion}

The SSPI method relies on three properties of the plasma behavior. First, a uniform propagation speed is assumed; second, the plasma is taken to be nearly force-free, so that dramatic changes in field structure are not expected; and third, the general expansion of the plasma is assumed to be small during the measurement interval. The SSPI provides a powerful means of gaining information about the magnetic topology from a single discharge.

It should be noted that the SSPI method resembles a technique used for measuring force-free magnetic fields in space plasmas. Burlaga ${ }^{3}$ analyzed data from an earth-orbiting spacecraft in order to characterize the field of interplanetary magnetic clouds. In Burlaga's case, a single magnetic probe is located on the spacecraft while the huge magnetic cloud propagates by it. Burlaga was able to successfully fit the data to a simple Lundquist $^{10}$ model $\left[B_{\theta}=B J_{1}(\lambda r), B_{z}\right.$ $=B J_{0}(\lambda r)$, and $\lambda$ uniform]. A key difference in our experiment is the availability of measurements at 12 positions transverse to the direction of motion of the plasma, allowing reconstruction of a plane of magnetic data rather than simply a single line.

\section{FLUX AMPLIFICATION}

In the coaxial gun formation method, helicity injection is achieved by driving poloidal current through the plasma, a process which can also be viewed as injection of toroidal flux to link pre-existing poloidal flux. This high helicitycontent plasma should then relax into a Taylor equilibrium. The total injected toroidal flux is typically substantially greater than the poloidal flux, ${ }^{11}$ whereas spheromak states contain comparable toroidal and poloidal fluxes. Thus, Taylor relaxation entails flux conversion which in this case of toroidal-flux rich injection would involve poloidal flux amplification accompanied by toroidal flux depletion. Indeed, this feature was first noted by Lindberg and Jacobsen ${ }^{12}$ and provides evidence of relaxation.

The camera and magnetic data in the previous section showed that the plasma in Regime II is qualitatively consistent with a spheromak configuration. This section presents evidence demonstrating that poloidal flux amplification, and thus Taylor relaxation, is taking place. That flux amplification is expected to occur in the present experiment can be shown as follows: With the gun operated under nominal settings in Regime II, the net toroidal injected flux can be found by time integrating the gun voltage (cf. Fig. 6) $\Phi_{\text {tor }}$ $=\int_{0}^{t} V_{\text {gun }} d t$. This calculation produces a net injected toroidal flux of $11 \mathrm{mWb}$ at the end of helicity injection $(9.5 \mu \mathrm{s})$, in comparison to the applied poloidal flux of $2 \mathrm{mWb}$.

\section{A. Poloidal flux calculation}

The poloidal flux function $\Psi$ is defined for an axisymmetric system as the net flux penetrating a circle of radius $r$ centered on the geometric axis and at axial position $z$

$$
\Psi(r, z)=\int_{0}^{r} 2 \pi r^{\prime} B_{z}\left(r^{\prime}, z\right) d r^{\prime} .
$$

The integration given in Eq. (2) can be straightforwardly applied to the magnetic data if the Single Shot Propagation Inference (SSPI) technique discussed in the previous section is used to convert the time coordinate into $z$ dependence. A sample calculation of the estimated poloidal flux function is presented in Fig. 14(a), corresponding to the magnetic data from the plasma discharge shown earlier in Fig. 8 (note: $t_{\text {magnetic_axis }}$ in Fig. 14(a) refers to the time at which the spheromak magnetic axis reaches the probe location). A constant is added to the integral to ensure that $\Psi\left(r=r_{\max }, z\right)$ $=0$. It can be seen from Fig. 14(a) that $\Psi(r, z)$ does not vanish at the geometric axis (i.e., at $r=0$ ) as would be expected; this is likely due to an offset in the device symmetry axis relative to the geometric axis. Unlike a fixed-boundary Taylor state, the expanding spheromak may be sensitive to the initial (i.e., formation) conditions, which may not be purely axisymmetric.

The location of maximum poloidal flux occurs at the spheromak magnetic axis. In this example, the peak value is approximately $4 \mathrm{mWb}$, or about twice the applied poloidal flux of $2 \mathrm{mWb}$. This amount of flux amplification is beyond what can be accounted for by uncertainties in the measurements or flux calculations, and constitutes strong evidence for Taylor relaxation in the form of reconnection. At axial locations $10-20 \mathrm{~cm}$ in front of or behind the magnetic axis (or equivalently, the peak of the poloidal flux), the flux function drops off dramatically. This is an indication of magnetic detachment from the gun.

It is also interesting to consider the poloidal flux time dependence. By calculating the maximum poloidal flux at various probe positions, the time evolution of a spheromak's poloidal flux can be examined. The result of such an analysis is shown in Fig. 14(b). Each point corresponds to 3-5 plasma shots taken with the magnetic probe located at a fixed axial position. The mean value of the maximum poloidal flux is shown, and the standard deviation of each calculation is indicated by the error bars. From this plot, it is evident that the poloidal flux rises from $2 \mathrm{mWb}$ up to $4 \mathrm{mWb}$ in the region $0<z<30 \mathrm{~cm}$, at which point it reaches a plateau. This region also comprises the interval in which X-point reconnection is taking place. The obvious interpretation is that poloidal flux amplification continues to occur during the 

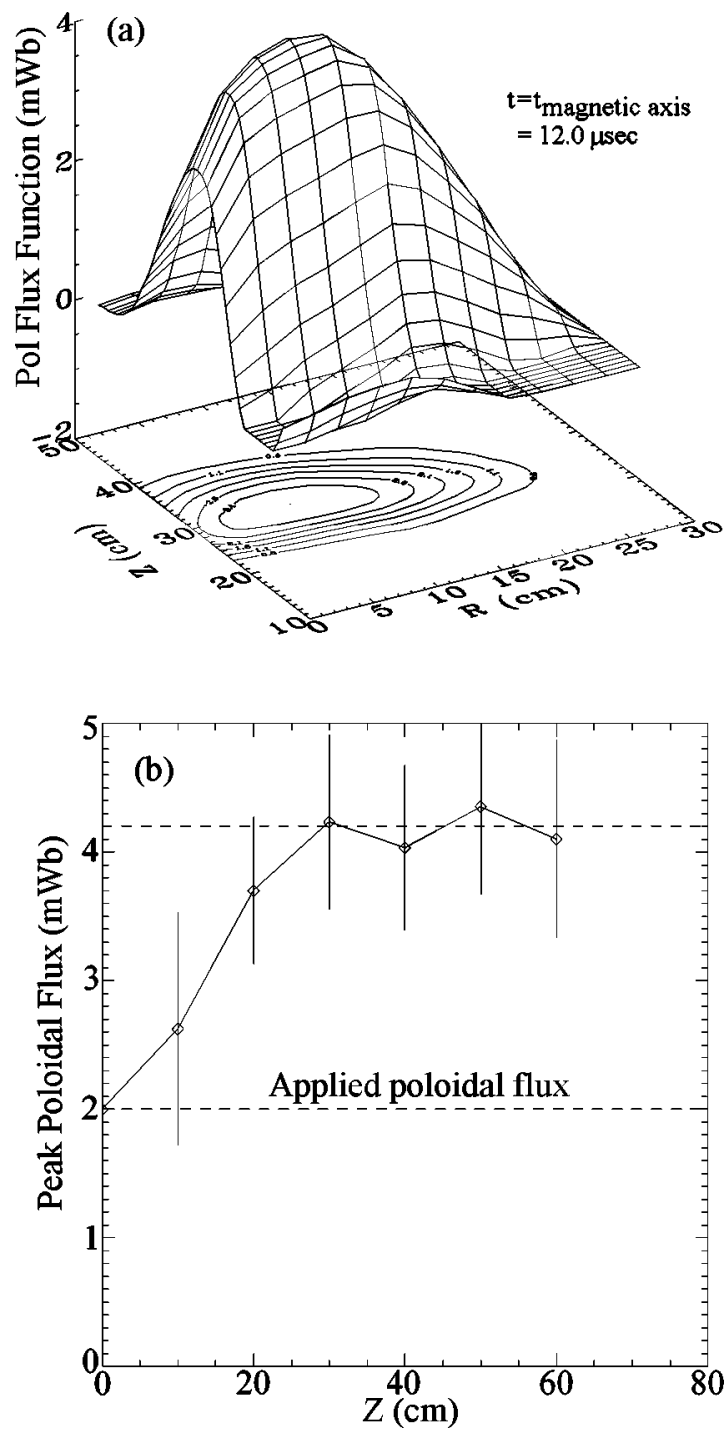

FIG. 14. (a) Calculated poloidal flux surface. (b) Maximum poloidal flux at sequence of probe positions.

reconnection-detachment process and terminates when the detachment is completed. Poloidal flux then achieves an equilibrium value and is thereafter conserved.

\section{B. Toroidal flux calculation}

The net toroidal flux contained within the spheromak can be defined as

$$
\Phi_{\text {tor }}=\int_{0}^{z_{\max }} d z \int_{0}^{r_{\max }} d r B_{\phi} .
$$

Calculation of this quantity presents some difficulty in the present situation because the field is only accurately known over a limited axial range in the vicinity of the probe. It is therefore necessary to restrict the integration area to a region encompassing the spheromak itself. For this reason, the integration area is defined as locations on the $(r, z)$ plane at which the toroidal magnetic field is greater than one half of its maximum value. This definition is essentially a Full Width at Half Maximum (FWHM) in two dimensions and as an example, the resultant region is highlighted in Fig. 15(a).
From this plot, it is apparent that the area is approximately equivalent to the area qualitatively encompassed by the vortex structure in the poloidal directions. However, the FWHM area appears to lag somewhat behind the vortex.

The result of this calculation at various probe positions is presented in Fig. 15(b). Again, each point corresponds to 3-5 plasma shots, and the error bars indicate the standard deviation. The toroidal flux decays from $\sim 11 \mathrm{mWb}$ down to $8 \mathrm{mWb}$ at $z=30 \mathrm{~cm}$, where it plateaus until a possible further dip at $z=60 \mathrm{~cm}$. Note that the reduction in toroidal flux occurs in the same interval over which the poloidal flux was observed to rise earlier. This behavior is consistent with the notion of a toroidal to poloidal conversion during the relaxation process.

In most spheromaks, the ratio of poloidal flux to toroidal flux is of order unity. In the present case, a ratio of $1: 2$ is observed in the plateau region: $30<z<60 \mathrm{~cm}$.

\section{Discussion}

The calculations of the poloidal and toroidal flux above lend credence to the hypothesis of spheromak creation. A significant $(2 \times)$ poloidal flux amplification effect has been observed, indicating that (i) Taylor relaxation is occurring and (ii) closed flux surfaces have been produced. The poloidal flux amplification approximately coincides with a corresponding drop in toroidal flux, as would be expected from the flux conversion principle.

The amount of amplification is somewhat less than the factor of five typically observed in earlier experiments; e.g., Ref. 13. However, the earlier experiments involved fluxconserver boundaries, which may influence the amplification process. Furthermore, reconnection is observed at probe positions as far out as $z=30 \mathrm{~cm}$, indicating that when the spheromak reaches this point, some closed field lines continue to be present. However, during this period the gun voltage is negative, possibly causing helicity to be extracted from the spheromak, thereby reducing the amplification factor.

The amplification occurs mainly within 3 gun radii $(30$ $\mathrm{cm}$ ) from the mouth of the gun, corresponding to 3.5-4 $\mu$ s of propagation time; a closed flux surface topology is observed through most of this propagation distance. This observation, coupled with the fact that the gun is no longer injecting helicity during this period indicates that the flux amplification must be produced entirely by relaxation (and not, e.g., helicity injection). Thus, it is natural to associate this time interval with the relaxation time, i.e., $\tau_{\text {relax }} \approx 3.5-4 \mu$ s.

\section{MEASUREMENT OF LAMBDA}

Taylor's theory ${ }^{1}$ predicts that an isolated plasma with finite helicity contained within flux conserving walls will relax into a force free state $\nabla \times \mathbf{B}=\lambda \mathbf{B}$ having $\lambda$ uniform throughout the volume. When such an equilibrium is attained, the plasma is in a minimum energy state for the given helicity.

Taylor's theory has been applied with considerable success to a wide variety of situations. However, it must be stressed that Taylor's theory assumes a fully relaxed plasma 


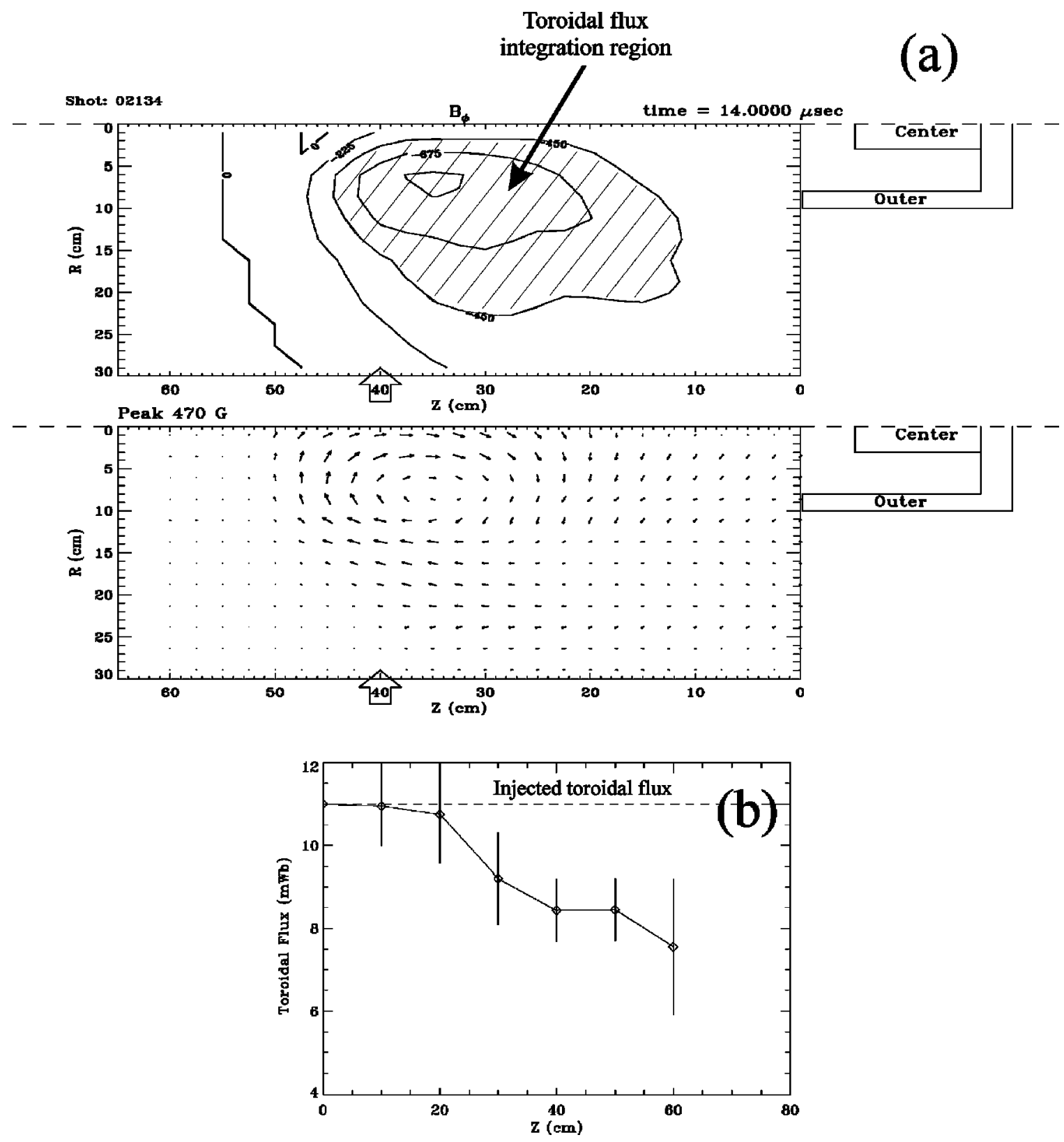

FIG. 15. (a) FWHM poloidal area in which toroidal flux is integrated. (b) Calculated toroidal flux (integrated within FWHM area) at sequence of probe positions.

and an essentially static environment. In real systems, the plasma may be influenced by the boundary conditions, helicity may not be perfectly conserved, and external sources may inject helicity. Any of these factors would compete with the mechanisms of Taylor relaxation and drive the plasma away from the minimum energy state. This section examines the extent to which the magnetic field structure in our experiment departs from a Taylor state, as evidenced by nonuniform $\lambda$.

\section{A. Gradients in $\lambda$ and helicity flow}

In analyzing magnetic configurations which differ from the minimum energy state, it is useful to consider the general class of plasmas which are in force-free equilibrium, but are not Taylor states. Taking the divergence of the force free relation $\nabla \times \mathbf{B}=\lambda \mathbf{B}$ shows that all force-free plasmas must satisfy the condition $\mathbf{B} \cdot \nabla \lambda=0$; i.e., $\lambda$ must be constant on a field line or, if there are flux surfaces, on a flux surface. Thus, for the general force-free plasma with poloidal flux surfaces, $\lambda$ may be written as $\lambda=\lambda(\psi)$, where $\psi$ is the poloidal flux function.

\section{B. Results}

The toroidal component of the current density in cylindrical coordinates is

$$
J_{\phi}=\frac{1}{\mu_{0}}(\nabla \times \mathbf{B})_{\phi}=\frac{1}{\mu_{0}}\left(\frac{\partial B_{r}}{\partial z}-\frac{\partial B_{z}}{\partial r}\right) .
$$

Evaluation of $\partial B_{r} / \partial z$ and $\partial B_{z} / \partial r$ is obtained in a poloidal 

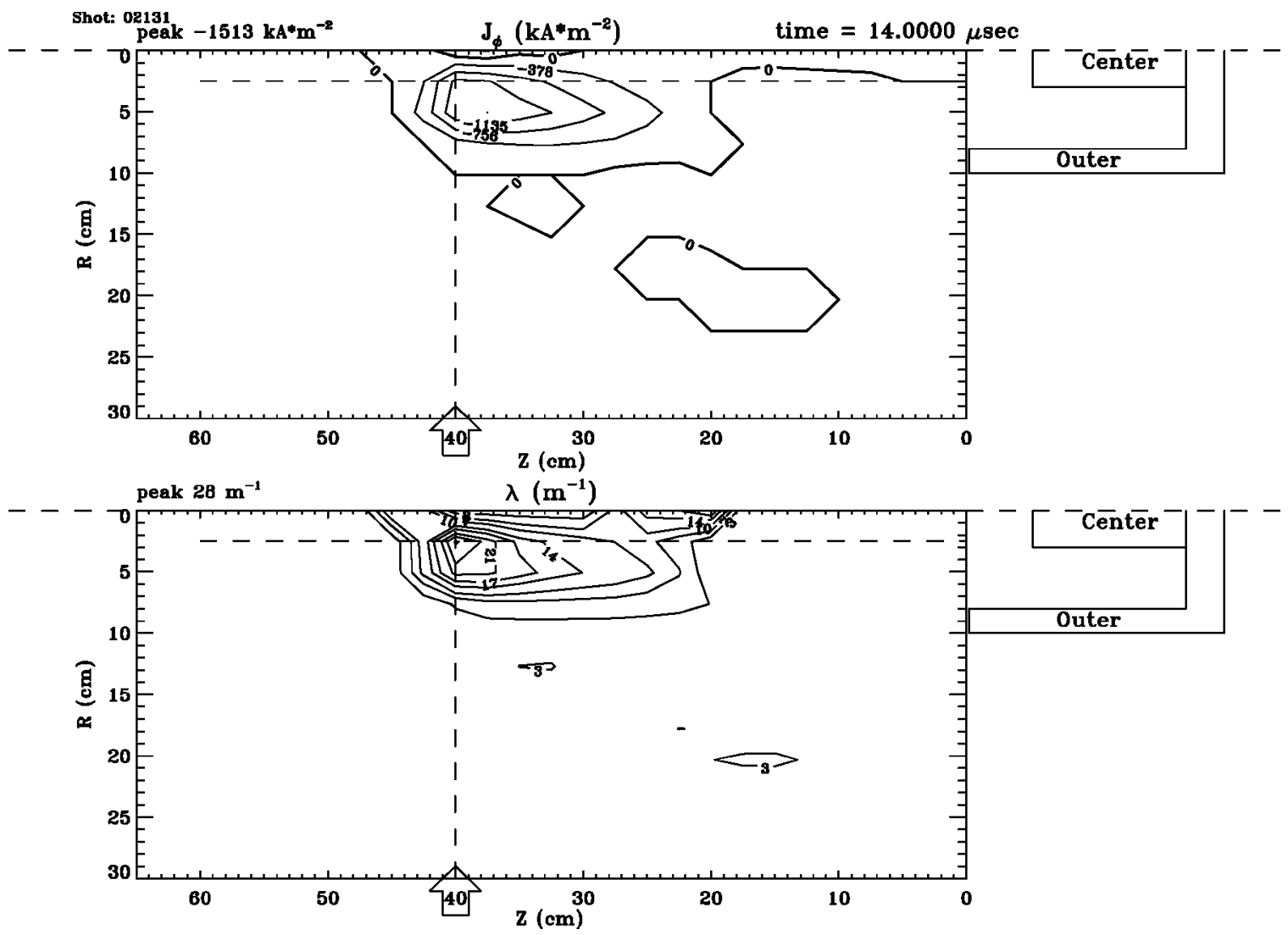

FIG. 16. Contour plots of calculated (a) toroidal current density and (b) $\lambda$. In both computations, SSPI method is used with magnetic probe located at $z$ $=40 \mathrm{~cm}$ and $t=14 \mu \mathrm{s}$.

plane employing the SSPI method, enabling the toroidal component of the current density to be calculated from a single plasma discharge.

\section{Spatial variation of $\lambda$}

Figure 16(a) shows a contour plot of $J_{\phi}$ in a poloidal cross section, calculated as described in the previous section. The magnetic probe was at $\phi=0, z=40 \mathrm{~cm}$ as indicated by the arrow in the horizontal axis. Note that the $B_{\phi}$ contours are similar to the $J_{\phi}$ contours as expected for a force-free configuration.

$\lambda$ can thus be quantitatively determined using $\lambda$ $=\mu_{0} J_{\phi} / B_{\phi}$. (This is similar to $\lambda$ calculations made from magnetograms of the solar surface. ${ }^{14}$ ) Contours of calculated $\lambda$ are shown in Fig. 16(b). It should be noted that, as $\left|B_{\phi}\right|$ $\rightarrow 0$, uncertainties in $\lambda$ grow without bound. For this reason $\lambda$ is set to zero at points where $B_{\phi}$ is less than $3 \%$ of its maximum value. Typically, $J_{\phi}$ is also small at these locations, so this should not significantly affect the results.

The dashed lines $(z=40 \mathrm{~cm}, r=2.5 \mathrm{~cm})$ intersect at the peak value of $\lambda$ of $28 \mathrm{~m}^{-1}$. Figure 17 shows an enlarged view near this region, as well as the radial and spatial profiles at $z=40 \mathrm{~cm}$ and $r=2.5 \mathrm{~cm}$, respectively. It is apparent that $\lambda$ assumes a centrally peaked distribution with FWHM of $\sim 5$ $\mathrm{cm}$. This centrally peaked $\lambda$ profile is consistent with a decaying spheromak.
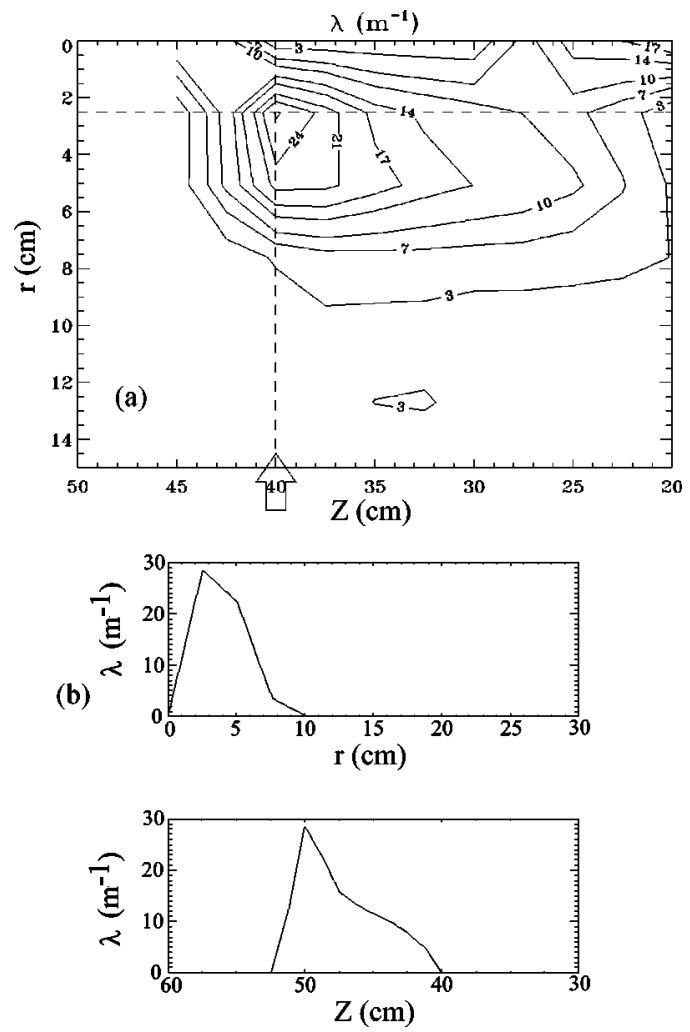

FIG. 17. (a) Closeup view of contour plot of $\lambda$. (b) Radial and axial profiles of $\lambda$, at $z=40 \mathrm{~cm}$ and $r=2.5 \mathrm{~cm}$, respectively. 


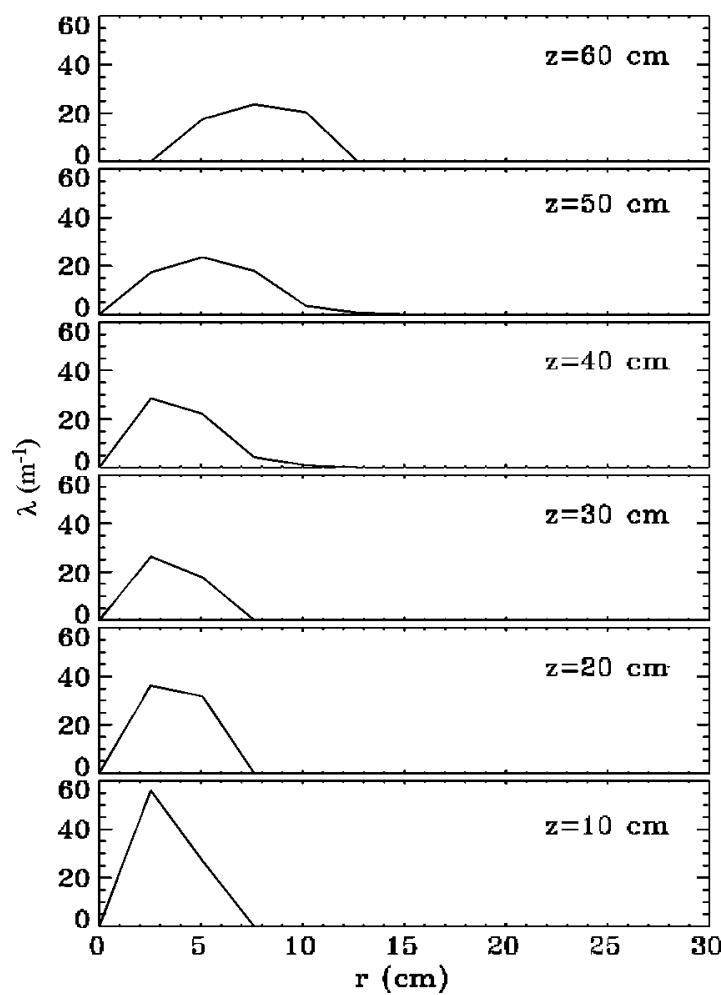

FIG. 18. Radial $\lambda$ profiles at axial sequence of probe locations.

\section{Time evolution of $\lambda$}

The time dependence of the $\lambda$ profile can be inferred from SSPI measurements at a sequence of axial locations. The data from such a scan is shown in Fig. 18. Each trace corresponds to the radial distribution of $\lambda$ at the magnetic axis (defined as the $z$ location with maximum $\lambda$ ). The spatial resolution of the calculation is limited by the magnetic probe array spacing; i.e., $2.5 \mathrm{~cm}$.

Two features are immediately evident. First, there is a definite broadening of $\lambda(r)$ at increasing distances from the gun. The FWHM increases from $\approx 4 \mathrm{~cm}$ at $z=10 \mathrm{~cm}$ to $\approx 8$ $\mathrm{cm}$ at $z=60 \mathrm{~cm}$. (This range in axial positions corresponds to a time interval of about $6 \mu \mathrm{s}$.)

Secondly, the peak values of $\lambda$ decrease with increasing z. This behavior is depicted in greater detail in Fig. 19(a). Each data point is obtained by averaging 3-5 shots at that axial location, and the variation in the values is indicated by the error bars. $\lambda$ decays from $\sim 55$ down to $\sim 25 \mathrm{~m}^{-1}$ as the plasma propagates away from the gun.

\section{Discussion}

It is evident from the plots in Fig. 18 that the spheromak exhibits roughly the same qualitative $\lambda$ profile regardless of probe axial location. $\lambda$ is always peaked near the magnetic axis and decreases monotonically away from the axis. This type of $\lambda$ gradient is consistent with that observed by Knox et al. ${ }^{15}$ during the decaying portion of the Compact Torus Experiment (CTX) spheromak.

Notably absent from any of these plots is an example of $\lambda$ peaking at the gun, which would be expected during spheromak sustainment. This nonobservation of hollow $\lambda$
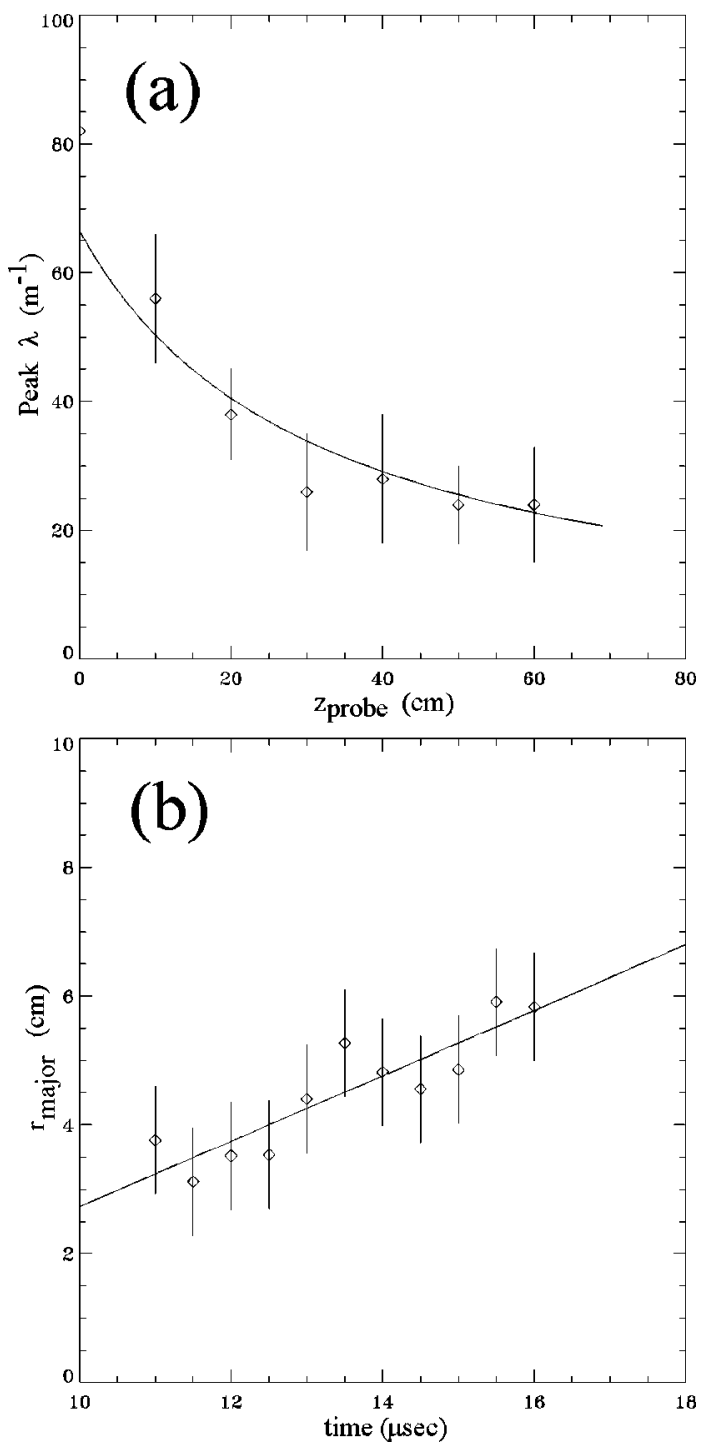

FIG. 19. (a) Peak values of $\lambda$ vs probe position. The solid curve represents a fit of the data to a self-similar expansion model. (b) Major radius of spheromak, as estimated from $\lambda$ profiles.

profiles is likely due to early detachment from the gun. When the magnetic probe is situated closest to the gun $(z=10 \mathrm{~cm})$, the spheromak magnetic axis arrives at the probe at $t=10$ $\mu \mathrm{s}$. This is after the end of the sustainment (i.e., helicity injection) phase of the discharge (cf. Fig. 6) at $9.5 \mu \mathrm{s}$.

In conventional spheromak experiments the plasma is injected into a flux-conserver and allowed to relax into an eigenstate prescribed by the flux conserver dimensions, so that $\lambda \rightarrow \lambda_{\text {geom }}$, where $\lambda_{\text {geom }}$ is the characteristic spatial dimension of the flux conserver. The distinguishing feature of the free expansion spheromak experiment discussed here is that the plasma never attains the true minimum energy state since this would entail filling the entire vacuum chamber. Thus, there is no predetermined $\lambda_{\text {geom }}$ value and the system scale length is simply the size of the expanding plasma. The results presented in the previous section indicate that the plasma $\lambda$ falls quickly from the gun value $\lambda_{\text {gun }}$ $=\mu_{0} I_{\text {gun }} / \Phi_{\text {bias }}=82 \mathrm{~m}^{-1}$ down to $\lambda=55 \mathrm{~m}^{-1}$ at $z=10 \mathrm{~cm}$.

This $\lambda$ decay is not a result of helicity dissipation be- 
cause it can be explained purely in terms of geometric expansion. One expects that the unbounded plasma and its embedded magnetic field would expand in a purely self-similar manner. In this simple prescription, the plasma would remain in a force-free state throughout the expansion. However, $\lambda$ would scale inversely with the system scale length; i.e., if the system doubles in size, $\lambda$ would fall to half its initial value. In order to test whether this phenomenon is occurring here, it is first necessary to determine the spheromak size.

Figure 19(b) shows a linear regression analysis of the plasma major radius, as estimated from the magnetic axis location; i.e., the peak of the radial $\lambda$ profile (cf. Fig. 18). Figure 19(b) indicates a uniform expansion rate. Assuming a self-similar expansion model

$$
\lambda=\frac{k}{r_{\text {major }}},
$$

with $k$ an undetermined parameter, and performing a leastsquares fit to the data in Fig. 19(a) gives $k=1.3$. With this value for $k$, the self-similar expansion model's predictions for $\lambda$ values are represented in Fig. 19(a) by the solid curve.

The fit is rather good, suggesting that the $\lambda$ decay is fully accounted for by plasma expansion, rather than helicity decay. This implies there is no significant helicity decay during the duration of these measurements. This would also imply that the resistive decay time $\tau_{\text {res }} \gg 7 \mu$ s, since helicity should decay on a resistive time scale.

\section{Relation to magnetic clouds}

Interplanetary magnetic clouds are regions within the solar wind with enhanced magnetic-field strength and a distinctly helical magnetic-field topology. The self-similar expansion described in the preceding discussion bear a striking resemblance to recent models of magnetic clouds. ${ }^{16}$ Rust and Kumar ${ }^{17}$ have proposed that magnetic clouds are associated with erupting solar prominences, which themselves have been shown to contain magnetic helicity and exhibit forcefree properties. ${ }^{18-21}$

In a recent magnetic cloud model proposed by Kumar and Rust, ${ }^{16}$ the magnetic cloud is modeled as a large aspect ratio torus in a uniform $\lambda$ force-free state. The field is described by the Lundquist solution within the plasma and the dipolar field of a current-carrying ring outside. Because of the toroidal current, a hoop force is generated in the radially outward direction, causing the structure to expand selfsimilarly. The model predicts a constant (rather than accelerating) rate of growth in both major and minor radii of the cloud. This behavior is borne out in observations. ${ }^{22}$ Using an argument similar to that presented in Sec. VIIC above, this expansion is accompanied by a reduction in $\lambda$. Since the total magnetic helicity $K$ is conserved, and $\lambda$ is proportional to the ratio $W / K$, the net magnetic energy $W$ must decay at the same rate as $\lambda$; i.e., Taylor relaxation requires magnetic energy dissipation as a result of the expansion. This dissipated magnetic energy will heat the magnetic cloud, a prediction consistent with observed high cloud temperatures. This prediction appears to conflict with the traditional thermodynamic concept of adiabatic cooling due to expansion.
In fact, both magnetic heating and adiabatic cooling are expected to occur, but heating will dominate for low $\beta$ plasmas.

Heating due to magnetic energy dissipation has previously been observed by Jarboe et al. ${ }^{23}$ on the CTX spheromak. This effect was found to heat the plasma up to $100 \mathrm{eV}$. However, the phenomenon producing the heating in that experiment was simple resistive decay. Both $W$ and $K$ decayed in amplitude while $\lambda$ remained constant.

\section{REGIME III: OPERATION WITH HIGH BIAS FLUX}

Based on the camera images, and the $\lambda_{\text {gun }}$ threshold dividing parameter space, it is natural to suspect that no closed flux surfaces are formed in Regime III and instead all field lines in the plasma begin and end at the gun electrodes.

For Regime III, an extensive scan was performed with the magnetic probe positioned in the ranges $10 \mathrm{~cm}<z<60$ $\mathrm{cm}$ and $-30^{\circ}<\phi^{\prime}<30^{\circ}$ (in probe and not gun coordinates), with increments of $\Delta z=5 \mathrm{~cm}, \Delta \phi^{\prime}=6^{\circ}$. Unlike Regime II, a high degree of reproducibility was achieved so that the SSPI method was not needed in Regime III.

Confirmation that a spheromak is not formed is provided by calculations of poloidal and toroidal fluxes. Computation of the poloidal flux must be performed in a manner different from the method described in Sec. VI because there is no magnetic axis to define the plane of integration. Instead, the net poloidal flux through an arbitrary $z=$ const. plane is found by numerically calculating the surface integral

$$
\Psi_{\mathrm{pol}}\left(z_{0}\right)=\int_{z=z_{0}} B_{z} d A_{-},
$$

where $d A_{-}$indicates that only negative polarity $B_{z}$ regions are included. (In principle, either positive or negative polarities can be counted, but not both. Negative polarity corresponds to the central column region.) Because only half the plane is diagnosed in the magnetic probe scan $(x>0)$, approximate bilateral symmetry is assumed and the computed half-plane surface integral is doubled. This constitutes a somewhat less restrictive assumption than the axisymmetry assumed above for the Regime II analysis.

Figure 20(a) shows a plot of the time dependence of the maximum value of the poloidal flux; i.e., at each time the flux is computed for all values of $z$ and the maximum is shown. This maximum value is generally found near the gun muzzle $(z=10 \mathrm{~cm})$. The curve peaks at $2 \mathrm{mWb}$ at $16 \mu \mathrm{s}$. Comparison with the applied $6 \mathrm{mWb}$ bias flux shows that no flux amplification is taking place. This indicates that most of the poloidal flux does not distend out of the gun sufficiently to penetrate the $z=10 \mathrm{~cm}$ plane; i.e., one gun radius away.

A plot of the time dependence of the total toroidal flux is shown in Fig. 20(b). In this case, the flux is computed by integrating $B_{\phi}$ over the entire surface of the natural poloidal plane $(\phi=0)$. This curve exhibits a similar time dependence to the poloidal flux and peaks at about $2.7 \mathrm{mWb}$. Comparison with the toroidal flux measured in Regime II suggests that most of the poloidal current (which generates the toroidal flux) is trapped within the gun. 

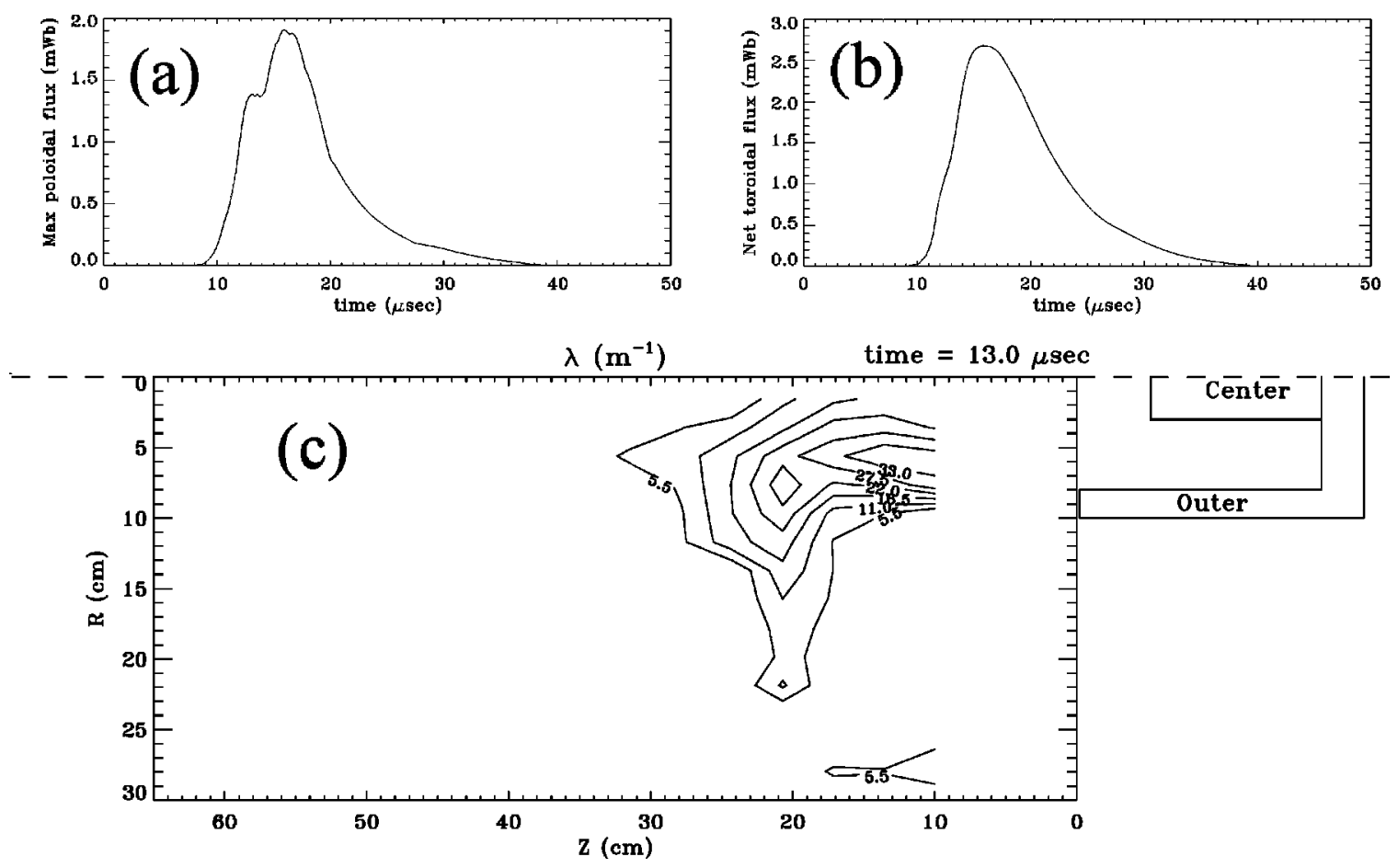

FIG. 20. (a) Maximum value of the poloidal flux in Regime III. Poloidal flux is computed from volumetric B-probe scan data over all $z$ planes; maximum value is shown. (b) Net toroidal flux vs time, computed using B-probe scan data, from the surface integral of $B_{\phi}$ over the poloidal plane $\phi=0$. (c) A contour plot of $\lambda$ at $t=13 \mu \mathrm{s}$, in the poloidal plane $\phi=0$, as computed from the magnetic probe scan data.

Finally, a calculation of the $\lambda$ profile is useful for determining the direction and magnitude of helicity flow. Figure 20(c) shows a contour plot of $\lambda$ in the natural poloidal plane at $t=13 \mu \mathrm{s}$. This shows that, unlike Regime II, there is no local $\lambda$ maximum propagating away from the gun. Instead, $\lambda$ monotonically decreases from the gun. At $t=13 \mu \mathrm{s}$, the maximum magnitude of $\lambda$ is $36 \mathrm{~m}^{-1}$, in comparison to $\lambda_{\text {gun }}=245 \mathrm{~m}^{-1}$. Thus, there is an extreme gradient in $\lambda$ at the mouth of the gun, providing confirmation that the majority of the helicity is locked up within the gun.

\section{SUMMARY}

Four distinct operational regimes were identified and correlated with $\lambda_{\text {gun }}$ values.

Data obtained with the gun operating in Regime II was consistent with the hypothesis of Taylor relaxation into an axisymmetric spheromak state. Magnetic data shows a striking vortex-like poloidal magnetic pattern showing that the plasma possesses the closed flux surface topology characteristic of an axisymmetric spheromak. Centrally peaked $\lambda$ profiles were observed, indicating that helicity flows from the magnetic axis; i.e., the spheromak is decaying. A broadening of the $\lambda$ profile with time implies expansion in the characteristic linear dimension. Analysis of the time dependence of the peak values of $\lambda$ suggests that a self-similar expansion model reasonably explains the $\lambda$ decay. The general characteristics of the expanding spheromak are similar to observations and models of magnetic clouds.

Photos and magnetic-field measurements in Regime III show that detachment of a configuration with closed magnetic topology does not occur. Analysis of poloidal flux, to- roidal flux, and $\lambda$ profiles indicate that the bulk of the magnetic flux and helicity do not emerge from the gun in Regime III because of excess bias flux; i.e., the plasma is unable to break off from the gun.

\section{ACKNOWLEDGMENTS}

This work was taken from the doctoral dissertation of one of the authors (J.Y.). The research was supported by U.S. Department of Energy Grant No. DE-FG0398ER54461. The ICCD camera was purchased in part by a Powell equipment grant. The authors wish to thank F. T. Cosso for technical assistance.

${ }^{1}$ J. B. Taylor, Phys. Rev. Lett. 33, 1139 (1974).

${ }^{2}$ J. B. Taylor, Rev. Mod. Phys. 58, 741 (1986).

${ }^{3}$ L. F. Burlaga, J. Geophys. Res. 93, 7217 (1988).

${ }^{4}$ T. R. Detman, M. Dryer, T. Yeh, S. M. Han, S. T. Wu, and D. J. McComas, J. Geophys. Res. 96, 9531 (1991).

${ }^{5}$ Y. Nakagawa, M. A. Raadu, D. E. Billings, and D. McNamara, Sol. Phys. 19, 72 (1971)

${ }^{6}$ M. B. Moldwin and W. J. Hughes, J. Geophys. Res. 99, 183 (1994).

${ }^{7}$ A. Konigl and A. R. Choudhuri, Astrophys. J. 289, 173 (1985).

${ }^{8}$ H. Alfvén, L. Lindberg, and P. Mitlid, J. Nucl. Energy, Part C 1, 116 (1960).

${ }^{9}$ T. H. Jensen and M. S. Chu, Phys. Fluids 27, 2881 (1984).

${ }^{10}$ S. Lundquist, Ark. Fys. 2, 361 (1950).

${ }^{11}$ T. R. Jarboe, Plasma Phys. Controlled Fusion 36, 945 (1994).

${ }^{12}$ L. Lindberg and C. Jacobsen, Astrophys. J. 133, 1043 (1961).

${ }^{13}$ W. C. Turner, G. C. Goldenbaum, E. H. A. Granneman, J. H. Hammer, C. W. Hartman, D. S. Prono, and J. Taska, Phys. Fluids 26, 1965 (1983)

${ }^{14}$ A. I. Pevtsov, R. C. Canfield, and T. R. Metcalf, Astrophys. J. Lett. 425, L117 (1994). 
${ }^{15}$ S. O. Knox, C. W. Barnes, G. J. Marklin, T. R. Jarboe, I. Henins, H. W. Hoida, and B. L. Wright, Phys. Rev. Lett. 56, 842 (1986).

${ }^{16}$ A. Kumar and D. M. Rust, J. Geophys. Res. 101, 15667 (1996).

${ }^{17}$ D. M. Rust and A. Kumar, Sol. Phys. 155, 69 (1994).

${ }^{18}$ H. Zirin and A. B. Severny, Observatory 81, 155 (1961)

${ }^{19}$ J. Heyvaerts and E. R. Priest, Astron. Astrophys. 137, 63 (1984).
${ }^{20} \mathrm{E}$. Tandberg-Hanssen, The Nature of Solar Prominences (Kluwer Academic, Dordrecht, 1995).

${ }^{21}$ P. M. Bellan and J. F. Hansen, Phys. Plasmas 5, 1991 (1998).

${ }^{22}$ V. Bothmer and R. Schwenn, Space Sci. Rev. 70, 215 (1994).

${ }^{23}$ T. R. Jarboe, C. W. Barnes, I. Henins, H. W. Hoida, S. O. Knox, R. K. Linford, and A. R. Sherwood, Phys. Fluids 27, 13 (1984). 